\title{
The Fontan Circulation: The Known, the Unknown and the Plausible
}

\author{
Jacoba J. Louw $\cdot$ Marc Gewillig
}

Published online: 1 March 2013

(C) Springer Science + Business Media New York 2013

\begin{abstract}
The Fontan circulation is used as palliative surgery in the functionally univentricular heart. Due to the advances made in prenatal diagnosis, cardiac surgery, technology, and monitoring in complex congenital heart disease, survival rates have improved significantly. For this group of patients, the physiology and pathology remain challenging and eluding, even more so in the long-term follow-up. In the last decade pulmonary vascular resistance has been shown to be of utmost importance regarding cardiac output both at rest and during exercise. The aim of this article is to review the most recently published data, namely new concepts and our current understanding regarding the physiology; performance during exercise; the value of imaging modalities; and chronic liver pathology in the Fontan circulation.
\end{abstract}

Keywords Fontan circulation - Univentricular heart . Single ventricle $\cdot$ Cardiopathy $\cdot$ Congenital heart disease Pulmonary vascular resistance

\section{Introduction}

In mammals, the cardiovascular system consists of a double-circuit, pulmonary and systemic, connected in series

J. J. Louw · M. Gewillig ( $ه)$

Pediatric and Congenital Cardiology,

University Hospitals Leuven, Herestraat 49,

3000 Leuven, Belgium

e-mail: marc.gewillig@uzleuven.be

J. J. Louw

e-mail: jacoba.louw@uzleuven.be and powered by a double pump. Many complex cardiac malformations are characterized by the existence of only one functional ventricle. At birth, in a functionally univentricular heart (UVH) the circulation cannot be connected in series but remains in parallel. This has two major disadvantages: arterial desaturation, both at rest and increasing during exercise, and a chronic volume overload to the single ventricle. This chronic volume overload will progressively impair the ventricular function, leading to a gradual attrition due to congestive heart failure from the third decade, with few survivors beyond the fourth decade.

In 1971, Fontan and Baudet [1] from Bordeaux, France, reported a new approach to the operative treatment of the functionally UVH by separating the systemic and pulmonary circulation in a staged method. Advantages of a Fontan circuit include (near-) normalization of the arterial saturation, and abolishment of the chronic volume overload. However, because the flow to the heart is hindered by the pulmonary impedance, the price for such a circulation includes chronic "hypertension" and congestion of the systemic veins, with decreased cardiac output both at rest and during exercise. Furthermore, the Fontan ventricle has a bad reputation: it is known to be large, hypertrophic, hypo-contractile, and non-responsive to the usual means utilized to increase cardiac output.

In recent years, our understanding of the physiology has changed considerably. We have learned that, once created, the Fontan circuit allows little modulation. The ventricle itself has become an "innocent bystander" and only propels the received amount of pulmonary blood flow forward into the systemic circulation. The control of preload, and therefore cardiac output, is essentially determined by the development of the pulmonary vascular impedance. Combined efforts are being made to improve the pulmonary vasculature, and accordingly, the long-term quality of life. 


\section{Surgical Approach: Can We Do Better?}

The functionally UVH comprise a morphologically heterogeneous group, and therefore different surgical strategies exists to obtain the best possible result for each individual patient. The jury is still out on the best course to follow and should be tailored to each specific patient.

The staged approach to creating a Fontan circulation allows the body to adapt progressively to the vastly different hemodynamic conditions, and to be corrected if indicated, thus reducing the overall operative morbidity and mortality. The current strategy, including performing total cavo-pulmonary connection (TCPC) at a younger age, has reduced the operative mortality to $<5 \%$ (compared to $15-30 \%$ initially) and a $85 \%$ survival at the age of 20 years [2]. The initial focus has been on the preservation of ventricular function. However, it has become clear that the pulmonary vasculature and pulmonary vascular resistance (PVR) are of the crucial importance in determining the final cardiac output and daily performance. Therefore, a difficult balance is necessary to sustain adequate volume load for sufficient time to allow optimal development and growth of the pulmonary vasculature before the Glenn shunt and the subsequent TCPC, but at the same time not overloading and damaging the ventricle [3]. The creation of a bidirectional cavo-pulmonary shunt (BCPS) which unloads the ventricle has an optimal result for ventricular function when performed at the age of 3-6 months [4].

Hybrid and Norwood procedures differ significantly in terms of stage II palliative procedures and growth of pulmonary vessels. When compared, in a short-term retrospective study of 75 infants, the survival rates were shown to be similar in these two groups, but there is a higher need for re-intervention of the pulmonary arteries to achieve satisfactory pulmonary artery growth in the hybrid group [5]. As explained before, this ultimately determines the long-term outcome.

An alternative option of mid-term palliation, i.e., BCPS with additional pulmonary blood flow, has shown a survival rate of $89 \pm 4 \%$ at 15 years. Additional pulmonary blood flow is achieved either by aorto-pulmonary shunt or through antegrade pulmonary outflow. In a recent retrospective study carried out in 65 patients, this strategy allowed postponement of the Fontan procedure with a median time of 7 years. Clinical failure occurred in 13 patients $(19 \%)$ [6]. In another study, this approach showed higher arterial oxygen saturations, larger mean body surface area (BSA), as well as a longer median interval before TCPC of 3.42 versus 2.9 years. This may theoretically be beneficial for the pulmonary artery growth needed for a successful TCPC and may ensure the insertion of a larger extracardiac conduit to avoid prosthesis-patient mismatch [7], but the superiority of the outcome in the long term still needs to be determined.

\section{Fontan Physiology, PVR and Exercise}

The Fontan circulation-even with an optimal result at every level-has a unique physiology with very specific features which are uncommon or even absent in the normal biventricular circulation. Typically the following aspects are found: an enlarged hypertrophic ventricle with increased afterload, decreased systolic and diastolic function, and a decreased stroke volume and cardiac output, both at rest and during exercise (Fig. 1). Many of these abnormalities are "secondary" to any Fontan circuit and not necessarily the primary cause for the low flow observed in the Fontan circuit. Known strategies aiming to "normalize" these secondary changes are very unlikely to yield a good clinical result, and will cause great frustration to the clinician. Interpretation of the numerous abnormal findings thus requires a profound knowledge of the Fontan physiology [8].

At birth, the single ventricle, having been responsible for the systemic, pulmonary, and placental circulation, will be large for BSA with compensated overgrowth, adapted to the volume overload. During the neonatal period, a volume overload persists for a few months due to increased pulmonary flow. By the time the Glenn state removes this volume load, the ventricle has developed overgrowth, dilation, and remodeling, many of which persist by the time the Fontan circuit is completed and thereafter. Due to the chronically reduced preload after Fontan connection there will be reduced ventricular growth, allowing the preloadventricular size mismatch to decrease. Therefore, the best

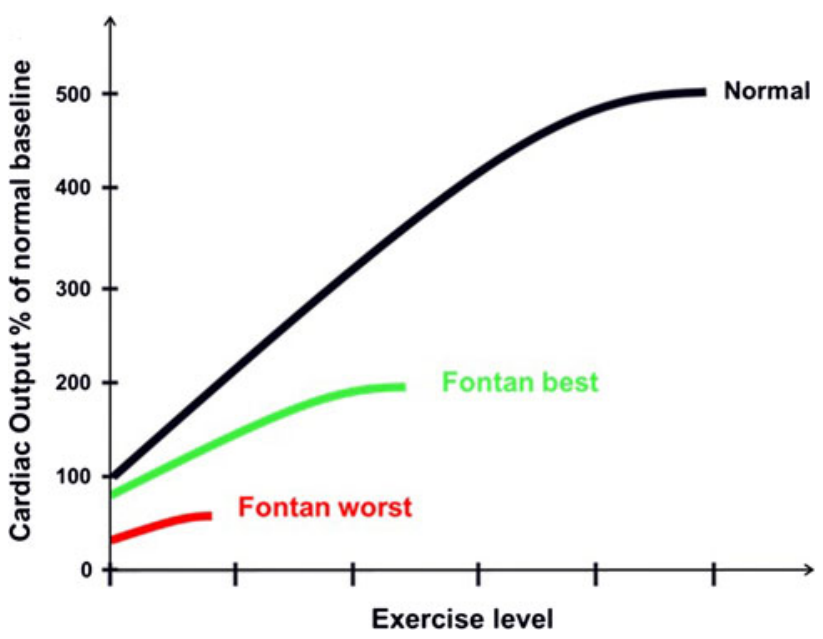

Fig. 1 Exercise and output: normal versus Fontan circulation: a normal subject with a biventricular circuit can increase his or her output by a factor of 5 (black line). In Fontan patients, output is significantly impaired both at rest and during exercise; at best (green line) the output is mildly decreased at rest, with moderate capacity to increase flow during (limited) exercise. At worst (red line), the output is severely reduced at rest and barely augments during exercise. Adapted from [8] (Color figure online) 
theoretical treatment is to avoid excessive volume overload, thereby avoiding dilation and overgrowth, and avoiding excessive unloading [9].

The surgical techniques have been optimized as far as the TCPC is concerned, and creating a TCPC increases maximal oxygen uptake $\left(\mathrm{VO}_{2}\right)$ by approximately $20-25 \%$. Nonetheless, these patients still remain below age- and sexmatched controls for maximal $\mathrm{VO}_{2}$ and have a blunted cardiac output response. Recent studies further report an annual decline of 1-3\% in maximal oxygen consumption starting at an adolescent age. The average peak consumption ranges from 19 to $28 \mathrm{ml} / \mathrm{kg} / \mathrm{min}$, or $50-60 \%$ of the predicted consumption for age [10].

In a normal biventricular circuit, PVRs and systemic vascular resistances are reduced during exercise. Additional pulmonary blood vessels, which are normally closed during rest, are being recruited, as well as active pulmonary arterial vasodilatation due to release of nitric oxide (NO). $\mathrm{NO}$ is released from the endothelium due to the increased flow and pulsatility. Fontan patients have insufficient pulmonary and systemic vasodilatation, most probably related to the non-pulsatile blood flow and systemic vascular endothelial dysfunction [11]. They are also less reactive to hyperemic and arterial vasodilation stimuli. This suggests that an abnormal systemic arterial endothelium-derived NO pathway exists $[12 \bullet \bullet]$.

Long-term follow-up has shown that PVR may rise many years after the Fontan procedure has been performed. Mechanisms of increased PVR might be micro-emboli, aging, obstructed airways caused by lymphatic dysfunction, lack of pulsatile pulmonary flow causing a release of endothelium-derived vasoactive molecules, and prolonged overexpression of vasoconstrictors such as endothelin-1. Mean plasma level of endothelin-1 has been shown to be significantly higher in Fontan patients compared to healthy controls.

The entity of decreasing PVR allowing better filling due to increased pulmonary blood flow has fuelled research in the recent treatment strategy of pulmonary vasodilators. These may improve exercise tolerance, but until now studies have shown little relevant effect.

Sildenafil is a phosphodiesterase 5-inhibitor that increases intracellular cyclic guanosine monophosphate and thus leads to a potent selective vasodilatory effect on the pulmonary vasculature. It is frequently used to reduce PVR in the pediatric population with pulmonary hypertension. In a recent randomized, double-blinded, placebocontrolled, crossover trial conducted in 28 Fontan subjects during 6 weeks, a significantly decreased respiratory rate and decreased minute ventilation was noted at peak exercise. Oxygen consumption during peak exercise showed no difference although there was a suggestion of improved oxygen consumption at the anaerobic threshold. An increase in exercise capacity was also documented in patients with single left or mixed ventricular morphology, as well as those with more advanced cardiac failure, i.e., baseline NBP level of $>100 \mathrm{pg} / \mathrm{ml}$ [13]. These findings suggest that sildenafil may be an important agent for improving exercise performance in children and young adults with single-ventricle physiology after the Fontan operation, but previous studies have shown small and inconsistent improvements in $\mathrm{VO}_{2}$ and in the ventilation to carbon dioxide production $\left(\mathrm{VCO}_{2}\right)$ [14].

The role of bosentan in the treatment of patients with a Fontan circulation is still unclear. In patients with pulmonary arterial hypertension (PAH), therapy with bosentan, an endothelin-1 receptor antagonist, has demonstrated to improve exercise capacity and to reduce the elevated PVR. An improvement in exercise capacity during treatment is predictable in patients with PAH using the baseline right ventricular contraction duration, which is not possible in Fontan patients [15]. No significant improvement in 6 min walking distance was seen in a small group of 10 failing Fontan patients treated with bosentan for 12 weeks. In another small study of eight patients, bosentan showed improvements in New York Heart Association (NYHA) functional class and systolic ventricular function after 6 months of treatment. However, the long-term effect of reducing the PVR by bosentan treatment on exercise capacity in Fontan patients, as well as safety and tolerability, remains unknown [16].

An additional way of increasing cardiac output was shown by Shafer et al. [17••]. In a normal biventricular circulation cardiac output is achieved by an increase in heart rate and a balanced increase in biventricular stroke volume. This is accomplished by different mechanisms, including the venous return via skeletal muscle contraction, or muscle pump, and the additional increase in venous return during inspiration (mostly by increased flow from the inferior vena cava), or ventilatory pump. On evaluating the muscle and ventilatory pumps in the univentricular circulation after Fontan repair the following was found in a small $(n=9)$, case-control study: the muscle pump contributed the most to stroke volume, $17 \%$ compared to $6 \%$ in controls $(p=0.06)$. There was no further significant increase $(<10 \%)$ in stroke volume with an increase in ventilatory rate (ventilatory pump) in either of the two groups. The muscle pump alone showed a significant increase in cardiac index of $30 \%$ (compared to $27 \%$ in controls). In submaximal exercise, a $\mathrm{VO}_{2}$ increase of $>50 \%$ greater was shown in controls compared to Fontan patients, while no difference was present at rest. Interestingly, the heart rate response was blunted in Fontan patients, being $70 \%$ higher in control patients compared to only $45 \%$ in Fontan patients. Not surprisingly, Fontan patients had lower $\mathrm{VO}_{2}$, cardiac index, and stroke volume 
with submaximal exercise. In this study, the detrimental effects of an increase in the thoracic pressure, with a decrease in the systemic venous return, and therefore cardiac output, was again demonstrated.

There is also considerable interest in development of implantable assist devices either as a "bridging" therapy to transplantation or as "destination therapy." Preliminary studies by computer simulation and mock-circulation with a catheter-based viscous impeller pump (VIP) to augment cavo-pulmonary flow and simultaneously reduce systemic vascular resistance as well as improve cardiac output shows promising results. A VIP augmented four-way TCPC flow and restored systemic pressures and flows to biventricular values with low hemolysis risk on pediatric scales [18].

\section{Value of New Imaging Modalities}

The utility of several new imaging modalities has been evaluated in the Fontan population. In 43 patients studied 1 month and 5 years after TCPC, radionuclide imaging was shown to be superior to angiocardiography in evaluating functional pulmonary perfusion [19]. Three-dimensional rotational angiography enhances diagnostic capabilities regarding visualization of the pulmonary arteries and veins due to their frequently encountered oblique course; a "cranial view" is required to see abnormalities in these vessels. In a study of 32 patients, additional information leading to nine interventions was detected. Furthermore, 22 lesions were "missed" by the currently used bi-plane angiography [20].

Quantifying systemic to pulmonary collaterals (SPC) by cardiac magnetic resonance before TCPC was shown to be reliably calculated by the difference between pulmonary venous return and pulmonary artery flow. As expected, an increased SPC flow was strongly associated with increased postoperative pleural effusion volume and duration, as well as prolonged intensive care unit and hospital stays. Preoperative risk factors, i.e., older age at $\mathrm{BCPC}$, higher oxygen saturations, reduced PA branch size, and absence of PA pulsatility, were associated with higher SPC flow. Most patients had small and multiple collateral vessels, which would make intervention of these vessels prior to TCPC aggressive and difficult [21].

\section{Liver Pathology}

The liver is undoubtedly put at risk in the Fontan circulation due to the chronically elevated venous pressure and decreased cardiac output. In a retrospective study done in 42 Fontan patients, the most common abnormalities detected by magnetic resonance imaging (MRI) or computed tomography examinations were abnormal parenchymal enhancement $(90 \%)$, hypervascular nodules $(31 \%)$, cirrhosis $(19 \%)$, and fibrolamellar hepatocellular carcinoma ( $2 \%)$. These lesions become more apparent as duration after TCPC increases, which might indicate underlying hepatic dysfunction and fibrosis [22].

Some centers recommend a prospective protocol for hepatic evaluation, including liver function tests, alpha-fetoprotein and clinical evaluation by a hepatologist, starting from 10 years after TCPC. Liver biopsy, although very invasive, seems to be the most reliable in detecting the degree of hepatic pathology, but results and its therapeutic relevance in the clinical setting still need to be correlated with the current imaging modalities [23].

\section{Can We Predict the "Failing" Fontan?}

It remains challenging and difficult to predict the "failing" Fontan. These patients have lived with less than ideal cardiac output their entire lives and thus will often fail to report or show progressive deterioration until a quite advanced stage. Frustratingly, conventional imaging, including echocardiography, angiography, and MRI, still disappoint in reliably detecting subtle changes before advanced stages. Regrettably, even though several advances have been made, we are still unable to treat a truly "failing" Fontan by means other than cardiac transplantation.

When a ventricle is large in a Fontan circuit, our management will differ whether we consider this a stretched small ventricle, or rather a collapsed under-loaded but overgrown pump. Similarly, when a Fontan ventricle is hypo-contractile, our management will differ whether we attribute this dysfunction to a damaged burned-out ventricle, or rather to under-filling of an overgrown and chronically deprived pump which requires more preload. Assessing the contribution of ventricular overgrowth, dilation, hypertrophy, dysfunction due to intrinsic myopathy, or dysfunction due to relative under-filling controlled by pulmonary vasculature, is very difficult. By necessity any analyses today will have to work with simplified and incomplete models.

Inherent to the Fontan circulation is the chronically elevated right atrial and vena caval pressure, as well as absence of a sub-pulmonary functional chamber. Thus, Fontan "failure" comprises different entities, including, but not limited to, the morphology, chronic venous congestion, and declining functional status. Intervention, either by percutaneous or surgical procedures, may improve or resolve some of these [2].

Brain natriuretic peptide (BNP) and N-terminal proBNP shows elevated levels before completion of TCPC or when patients were already symptomatic. A clear association was shown with NYHA functional class. BNP levels in asymptomatic Fontan patients were shown to be 
comparable to healthy controls. This can partly be explained by the "unloading" of the ventricle at TCPC, as BNP was shown to correlate with ventricular volume load. BNP assessment might therefore contribute to the evaluation, but cannot "predict" failure [24].

\section{Long-Term Outcome}

The Fontan circuit has known numerous modifications. Initially valves (cavo-atrial, atrio-ventricular, or -pulmonary) and different connections between the right atrium and the pulmonary artery (anterior atrio-pulmonary connection, with or without inclusion of a small hypoplastic right ventricle, or posterior atrio-pulmonary connection), as well as different materials (valved conduits, homografts, patches, or direct anastomosis) were used. The surgical techniques were suboptimal, which was reflected by the high need, reaching $40 \%$ in some series, for early and multiple revisions. Most of the older circuits are no longer created and considered obsolete; however, many patients still survive on such circuits. Thus, when assessing a patient with a Fontan circuit, the cardiologist needs to know exactly which type of surgical technique and what type of material was used in its creation.

There are several reasons why adult patients with a Fontan circuit do not represent the outcome of the current cohort of younger patients:

- The longest survivors of a Fontan operation, currently adults, were frequently not ideal candidates for this type of surgery, with many significant residual lesions related to the cardiac malformation and palliative procedures.

- A shunt procedure in the 1960s until 1980s was evaluated by its long-term relief of cyanosis. The fact that this shunt could induce mild pulmonary vascular disease, ventricular dysfunction, or pulmonary artery distortion was not-as it is now-the principal preoccupation of the surgeon.

- Previously, many centers have attempted to determine which combination of criteria could be ignored without compromising a "successful" outcome. Fontan circuits have at that time been performed on patients who at present would probably not be considered for such repair.

At present, patient selection prior to Fontan is more strict and precise. Early operative mortality has vastly decreased. There is a heightened awareness and more and improved imaging possibilities to detect residual lesions as well as more aggressive management thereof where possible. The success of a shunt is currently evaluated by an acceptable relief of cyanosis without a significant volume overload, by its induction of pulmonary growth without a negative effect on the pulmonary vasculature (including the period of growth after Fontan repair), and by allowing the patient to reach an age on which a Fontan circuit can be safely created. Excessive ventricular dilation, spherical reconfiguration, cardiac overgrowth, ventricular dysfunction, and mild pulmonary vascular disease or distortion are now rarely seen. The suboptimal candidates from the previous series who survived the operation unmistakably belong to the worst end of the clinical spectrum of results after Fontan correction, and will blur many reports on longterm results. We expect the current "better" circuits to have a "life-time" potential.

In some cases, therapeutic options are limited to cardiac transplantation. Cardiac transplantation in Fontan patients shows a higher early operative mortality of approximately $30 \%$ when compared with other forms of congenital heart disease. This can be attributed to multiple previous surgical interventions, preformed antibodies to donor leucocyte antigens (HLA), malnourishment, acute graft failure, and a complex venous anatomy as well as the consequences of increased PVR. Nonetheless, there seems to be no difference in the long-term survival, with survival rates of $54 \%$ 10 years post-transplant $[25,26]$.

\section{Conclusions}

Many studies have been conducted and various questions have been raised since the first Fontan circulation was reported in 1971. The surgeons have learned how to avoid cardiac factors that are deleterious for the Fontan circuit: ventricular overgrowth, hypertrophy, dilation and dysfunction; excessive unloading at the time of the cavo-pulmonary connection; and energy loss at the cavo-pulmonary anastomosis. Some improvement is still possible, but it has now become the exception that failure of the Fontan circuit is attributable to the surgical connection or primary cardiac dysfunction.

As mentioned before, once created, the Fontan circuit allows little modulation. With preserved ventricular function, the main determinant of cardiac output in the Fontan circuit has become the trans-pulmonary flow, or preload. Therefore, we should continue to focus on growth and optimal development of the pulmonary vasculature before Fontan surgery: the initial palliation is crucial, allowing adequate volume load for sufficient time to obtain optimal catch-up growth and development of the pulmonary vasculature before the Glenn shunt. Medical means of decreasing the PVR with use of medication has thus far been disappointing and of little functional relevance. 
Disclosure Jacoba J. Louw and Marc Gewillig declares no conflicts of interest.

\section{References}

Papers of particular interest, published recently, have been highlighted as:

-• Of major importance

1. Fontan F, Baudet E. Surgical repair of tricuspid atresia. Thorax. 1971;26(3):240-8.

2. Deal BJ, Jacobs ML. Management of the failing Fontan circulation. Heart. 2012;98(14):1098-104.

3. Gewillig M, et al. Volume load paradox while preparing for the Fontan: not too much for the ventricle, not too little for the lungs. Interact Cardiovasc Thorac Surg. 2010;10(2):262-5.

4. Cleuziou J, et al. Bidirectional cavopulmonary connection without additional pulmonary blood flow in patients below the age of 6 months. Eur J Cardiothorac Surg. 2008;34(3):556-61; discussion $561-2$.

5. Baba K, et al. Hybrid versus Norwood strategies for single-ventricle palliation. Circulation. 2012;126(11 Suppl 1):S123-31.

6. Gerelli $\mathrm{S}$, et al. Bidirectional cavopulmonary shunt with additional pulmonary blood flow: a failed or successful strategy? Eur J Cardiothorac Surg. 2012;42(3):513-9.

7. van Slooten YJ, et al. The effect of additional pulmonary blood flow on timing of the total cavopulmonary connection. Ann Thorac Surg. 2012;93(6):2028-33.

8. Gewillig M, et al. The Fontan circulation: who controls cardiac output? Interact Cardiovasc Thorac Surg. 2010;10(3):428-33.

9. Gewillig M, Kalis N. Pathophysiological aspects after cavopulmonary anastomosis. Thorac Cardiovasc Surg. 2000;48(6): 336-41.

10. Diller GP, et al. Predictors of morbidity and mortality in contemporary Fontan patients: results from a multicenter study including cardiopulmonary exercise testing in 321 patients. Eur Heart J. 2010;31(24):3073-83.

11. Inai $\mathrm{K}$, et al. Skeletal muscle hemodynamics and endothelial function in patients after Fontan operation. Am J Cardiol. 2004;93(6):792-7.

12. • Ciliberti P, Schulze-Neick I, Giardini A. Modulation of pulmonary vascular resistance as a target for therapeutic interventions in Fontan patients: focus on phosphodiesterase inhibitors. Future Cardiol. 2012;8(2):271-84. A thorough and clear explanation of the role of pulmonary vascular resistance in the Fontan circulation.

13. Goldberg DJ, et al. Impact of oral sildenafil on exercise performance in children and young adults after the Fontan operation: a randomized, double-blind, placebo-controlled, crossover trial. Circulation. 2011;123(11):1185-93.

14. Giardini A, et al. Effect of sildenafil on haemodynamic response to exercise and exercise capacity in Fontan patients. Eur Heart J. 2008;29(13):1681-7.

15. Schuuring MJ, et al. Rationale and design of a trial on the role of bosentan in Fontan patients: improvement of exercise capacity? Contemp Clin Trials. 2011;32(4):586-91.

16. Bowater SE, et al. The safety and effects of bosentan in patients with a Fontan circulation. Congenit Heart Dis. 2012;7(3):243-9.

17. $\bullet$ Shafer KM, et al. The importance of the muscle and ventilatory blood pumps during exercise in patients without a subpulmonary ventricle (Fontan operation). J Am Coll Cardiol. 2012;60(20):2115-21. A very interesting article explaining an additional way of increasing cardiac output in Fontan patients.

18. Giridharan GA, et al. Performance evaluation of a pediatric viscous impeller pump for Fontan cavopulmonary assist. J Thorac Cardiovasc Surg. 2013;145(1):249-57.

19. Wang $\mathrm{H}$, et al. The mid-term follow-up of pulmonary perfusion in patients after extracardiac total cavopulmonary connection. Nucl Med Commun. 2012;33(2):148-54.

20. Berman DP, et al. The use of three-dimensional rotational angiography to assess the pulmonary circulation following cavopulmonary connection in patients with single ventricle. Catheter Cardiovasc Interv. 2012;80(6):922-30.

21. Odenwald T, et al. Systemic to pulmonary collateral blood flow influences early outcomes following the total cavopulmonary connection. Heart. 2012;98(12):934-40.

22. Wallihan DB, Podberesky DJ. Hepatic pathology after Fontan palliation: spectrum of imaging findings. Pediatr Radiol. 2013; 43(3):330-8.

23. Rychik J, et al. The precarious state of the liver after a Fontan operation: summary of a multidisciplinary symposium. Pediatr Cardiol. 2012;33(7):1001-12.

24. Eindhoven JA, et al. The usefulness of brain natriuretic peptide in complex congenital heart disease: a systematic review. J Am Coll Cardiol. 2012;60(21):2140-9.

25. Mitchell MB, et al. Evidence of pulmonary vascular disease after heart transplantation for Fontan circulation failure. J Thorac Cardiovasc Surg. 2004;128(5):693-702.

26. Mitchell MB, Campbell DN, Boucek MM. Heart transplantation for the failing Fontan circulation. Semin Thorac Cardiovasc Surg Pediatr Card Surg Annu. 2004;7:56-64. 